\title{
«Women's Studies » aux Etats-Unis : trente ans de succès et de contestation
}

\section{Marilyn Boxer}

\section{(2) OpenEdition}

1 Journals

\section{Édition électronique}

URL : https://journals.openedition.org/clio/142

DOI : 10.4000/clio.142

ISSN : 1777-5299

Éditeur

Belin

Édition imprimée

Date de publication : 1 avril 2001

Pagination : 211-238

ISBN : 2-85816-577-7

ISSN : 1252-7017

Référence électronique

Marilyn Boxer, « "Women's Studies » aux Etats-Unis : trente ans de succès et de contestation », Clio. Histoire, femmes et sociétés [En ligne], 13 | 2001, mis en ligne le 14 novembre 2006, consulté le 23 avril 2022. URL : http://journals.openedition.org/clio/142 ; DOI : https://doi.org/10.4000/clio.142

Ce document a été généré automatiquement le 23 avril 2022.

Tous droits réservés 


\title{
« Women's Studies » aux Etats- Unis : trente ans de succès et de contestation
}

\author{
Marilyn Boxer
}

\section{NOTE DE L'ÉDITEUR}

Marilyn J. Boxer analyse dans cet article la naissance et l'évolution des women's studies aux États-Unis et leur diversification actuelle, en particulier à travers leurs dimensions institutionnelles, professionnelles et idéologiques. En historienne restée attachée à la dénomination originelle des women's studies, elle aborde notamment le débat sur les gender studies, très mal connu en France. Nous espérons le prolonger par d'autres contributions d'historiennes.

1 En 1971, quand j'ai proposé mon premier cours de women's studies dans un institut universitaire de Californie du Sud, la première question à laquelle j'ai dû répondre fut : «Y a-t-il assez de matière pour tout un cours?» Quatre ans plus tard, quand j'ai demandé l'approbation d'une option women's studies auprès d'une commission universitaire, la question que l'on me posa fut : «Les women's studies constituent-elles une discipline? ? Grâce à plus de 10000 diplômes de troisième cycle portant sur une recherche en women's studies décernés entre 1978 et 1995, la première question ne se pose plus. La deuxième question revient encore fréquemment, et prend une résonance particulière dans le récent contexte qui tend à créer des filières doctorales interdisciplinaires qui soient des programmes en soi, et non confinés à «l'étude des femmes » (study of women) dans le cadre des disciplines traditionnelles. Le succès s'est accompagné d'une contestation croissante portant sur cette distinction, ainsi que sur l'emploi de «femmes » comme concept fondateur de la recherche féministe.

2 Depuis trente ans, le succès des women's studies en a surpris plus d'un dans l'enseignement supérieur aux États-Unis ${ }^{1}$. À l'opposé des principaux courants 
intellectuels et institutionnels du dernier demi-siècle qui évoluent vers des méthodes scientistes et analytiques, et tendent à éliminer, de façon concomitante, les questions des valeurs, les women's studies les placent au centre du débat. À l'encontre des tendances allant vers la spécialisation disciplinaire, l'isolement des départements universitaires et le divorce entre enseignement et recherche, les women's studies recherchent l'intégration. Elles sont maintenant établies dans plus de la moitié des universités américaines et recouvrent un grand éventail de programmes de deuxième et de troisième cycles, de centres de recherche, de journaux universitaires, d'associations professionnelles.

3 Pour comprendre ce développement, il est nécessaire d'avoir quelques notions sur l'histoire de l'accès des femmes américaines à l'enseignement supérieur, sur l'enseignement supérieur américain en lui-même, ainsi que sur l'histoire de la « seconde vague » du féminisme. Le succès est aussi le reflet de l'acceptation croissante de l'interdisciplinarité (en dépit d'une spécialisation croissante) et la visibilité grandissante de populations auparavant socialement marginalisées, dont les femmes, dans les universités et la société. De nombreux universitaires américaines mènent aujourd'hui des recherches et donnent des cours qui seraient inconcevables sans les mouvements pour les droits civiques des minorités, pour l'accès des étudiants au pouvoir, et pour la libération des femmes.

Bien qu'il soit impossible dans ce court essai de faire plus que d'évoquer quelques points essentiels de ce phénomène complexe et en perpétuel changement, je vais essayer de fournir quelques données utiles et d'éclairer certaines des questions qui affectent la recherche féministe dans son institutionnalisation, y compris le récent mouvement pour combiner ou subsumer les women's studies sous la rubrique des gender studies ou des études sur la sexualité. Je vais également essayer de me faire l'écho de l'argument selon lequel les women's studies semblent aujourd'hui, malgré leurs nombreux succès, être en situation périlleuse, à l'entrée d'un nouveau millénaire qui manque de fondation intellectuelle cohérente ${ }^{2}$.

Une croissance formidable

5 Commençons par les succès. Chaque année, d'après les sondages menés à la Graduate School of Education and Information Studies à l'université de UCLA, un nombre toujours plus grand d'universitaires déclare étudier les femmes (et le genre) et incorporer ce nouveau champ d'étude dans les cours. Dans un petit nombre de cas, ceci est le reflet de l'adoption par les commissions universitaires chargées des programmes d'une exigence de diversité qui inclut les women's studies dans la filière générale; les étudiants doivent s'inscrire à un ou deux cours mettant l'accent sur les femmes, les groupes raciaux et/ou les différences culturelles. En revanche, et plus communément, les cours sur l'histoire des femmes, la littérature féminine ou autres sont ajoutés aux listes des options proposées aux étudiants. Dans une plus large mesure, néanmoins, cela reflète les effets d'une recherche florissante; en 1978, l'organisme Dissertation Abstracts International a adopté les women's studies comme catégorie pour classifier les thèses de doctorat. Le total de cette année fut de 10. En 1995, le chiffre correspondant fut 2478 , et le total cumulé 13084 , dont 10278 représentaient des diplômes de doctorat et les autres des maitrises ${ }^{3}$.

6 L'intérêt du corps enseignant a à la fois suivi et renforcé celui des étudiants. Dès 1993, plus de $12 \%$ des étudiants avaient suivi des cours dans les women's studies, chiffre supérieur à tout autre champ interdisciplinaire ${ }^{4}$. Dans une époque marquée par une 
tendance sociétale insidieuse associant les études supérieures à la préparation d'une carrière professionnelle, les women's studies ont fourni un contrepoids important et ont permis ainsi de renforcer les disciplines littéraires et les sciences humaines qui souffraient du déclin de leurs effectifs. Elles ont aussi constitué un moyen supplémentaire (ou même alternatif) de diversifier des campus dévoués, tout au moins officiellement, à la cause de l'affirmative action. Les women's studies semblaient se conformer à l'esprit, sinon à la lettre, des exigences imposées par le titre IX de la loi fédérale de 1972 qui exigeait des universités recevant des fonds fédéraux qu'elles assurent un traitement égal des femmes et des hommes 5 .

$7 \mathrm{Au}$ début, le rôle des étudiant-e-s, en deuxième comme en troisième cycle, fut déterminant. La défense des femmes sur les campus a émergé de la participation aux mouvements pour les droits civiques, pour le pouvoir estudiantin et pour la libération des femmes. L'investigation universitaire a bientôt suivi la prise de conscience que l'on savait très peu de choses sur les femmes et que leur histoire - comme le reste - était encore cachée. Véritable mouvement d'épanouissement personnel, le besoin, et même la passion d'étudier les femmes se répandit rapidement depuis ses points de départ dans des villes de l'Est, l'Ouest, le Midwest et le Sud (incluant Seattle, New York, Chicago et La Nouvelle Orléans) pour apparaître sur tout le territoire. Le premier programme intégré d'études commença à San Diego State University (SDSU) en Californie, avec dix cours, un petit bâtiment spécifique, un budget de fonctionnement et 1,5 postes de professeurs titulaires. Inauguré officiellement comme un «programme de women's studies » au semestre d'automne 1970 (bien que cinq cours aient été offerts le printemps précédent), c'était en fait quasiment un département, ayant ses propres cours et une directrice de programme rendant ses comptes directement au viceprésident des études. Placé sous l'autorité d'un doyen d'université dès qu'une réorganisation fut opérée, ce programme ne fit jamais partie d'un autre département (point important pour le développement des women's studies à SDSU). Ce programme fit l'objet d'une attention nationale positive presque immédiatement, dans un article publié par Newsweek sur «l'une des nouveautés les plus en vue de l'enseignement supérieur ${ }^{6} »$.

8 L'Université de Cornell, dans l'État de New York, à l'origine au début de 1970 d'une série de conférences sur "l'évolution de la personnalité féminine ", lança le second programme. Ces deux programmes devinrent 150 en 1975, 300 en 1980, 450 au milieu des années 80, 600 en 1990, et aujourd'hui plus de 700. S'inspirant du puissant modèle des premiers programmes d'études de la condition des Noirs en Californie et dans l'État de New York, et d'Études des Américains d'origine mexicaine (ou Chicanos) en Californie, ces programmes émanaient principalement d'établissements d'enseignement supérieur, et différaient ainsi de modèles nationaux où les women's studies prospéraient davantage dans un cadre local militant et dans des centres de recherche. L'importance de l'enseignement dans le système des universités publiques américaines, dont les budgets dépendent des effectifs, donna du pouvoir aux étudiants qui «votaient avec leurs pieds", s'inscrivaient en grand nombre dans des classes de women's studies, souvent aux dépens de cours plus traditionnels. Pendant les années 1980, l'ère Reagan, les effectifs des écoles de commerce se multiplièrent, alors que les spécialisations dans les lettres diminuèrent. Des déclins vertigineux des effectifs dans les départements d'histoire et de littérature à cette époque autorisèrent les doyens, s'ils le désiraient, à attribuer des budgets discrétionnaires ${ }^{7}$. En tant que directrice des 
women's studies à SDSU entre 1974 et 1980, j'ai dirigé la croissance du département, passant de 3,4 à 8,1 postes d'enseignants.

Dès 1977, nous avions aussi obtenu l'approbation de huit cours de women's studies dans un programme d'éducation générale qui offrait relativement peu de choix aux étudiants. Notre cours, "Sexisme et sciences Sociales", décrit comme une "critique féministe des concepts et modes de pensée conventionnels et partiaux dans les disciplines traitant des rapports humains ", fut accepté comme introduction de base à une discipline, au même titre que l'Introduction à l'anthropologie, l'Introduction à la sociologie, et les Principes d'économie, mais seulement après une bataille dans le Sénat de l'université contre l'accusation portée par un anthropologue selon laquelle les women's studies étaient partiales, à la différence de sa discipline qui était, selon lui, exempte de présupposés. Tirant mes arguments de la sociologie du savoir et parlant pour notre département - utilisant «les outils du maître »-j'ai pu convaincre les autres membres du Sénat qu'aucune science sociale n'était impartiale.

Si l'on observe le développement des women's studies dans le réseau d'universités d'État en Californie (le plus grand réseau d'enseignement supérieur en quatre ans aux États-Unis), où dès 1972 des programmes de women's studies avaient été lancés depuis San Diego, tout au sud, jusqu'à Humboldt tout au nord, et où des programmes novateurs apparurent bientôt à San Francisco (qui incorporaient la diversité « raciale » dès le début), Sonoma (où la célébration d'une Journée nationale de l'histoire des femmes fut lancée), Sacramento (qui en 1973 accueillit la première conférence nationale), ce qui marque le plus n'est pas son ampleur géographique horizontale, mais plutôt sa structure de classe verticale. Les études supérieures aux États-Unis sont souvent remarquées pour leur pluralisme, leur diversité et décentralisation, qui expliquent en partie la prolifération rapide des women's studies. Ce que l'on remarque moins souvent, c'est le système de classe hiérarchique qui concentre une grande quantité d'étudiants dans les établissements de second rang, moins onéreux, alors que les meilleures universités, où la recherche est l'objectif principal, sont richement subventionnées. Le résultat est qu'en Californie, l'État le plus peuplé, les women's studies se développèrent bien plus lentement à la prestigieuse Université de Californie qu'à la California State University, parfois qualifiée d'» université du peuple». À ce jour, alors que l'Université de Californie abrite des centaines de chercheurs qui se consacrent à «l'étude des femmes " (study of women), elle attribue des ressources minimales à des postes d'enseignants désignés comme "women's studies». Des universités de recherche dans d'autres États, notamment dans le Midwest, ont investi bien plus lourdement. Des campus prééminents dans l'Illinois, l'Iowa, le Michigan, le Minnesota, l'Ohio et le Wisconsin ont tous établi de grands centres d'études féministes, et plusieurs ont récemment rejoint les rangs des universités offrant des diplômes de doctorat en women's studies.

Les facteurs institutionnels favorisant le succès

11 Aux États-Unis, la symbiose de l'enseignement et de la recherche dans les études supérieures permet aux professeurs d'enseigner dans leur domaine de recherche. Pendant les dix premières années de la croissance rapide des women's studies, les choix des étudiants avaient un grand poids dans les décisions budgétaires. Sur de nombreux campus, les women's studies naquirent des exigences des étudiant-e-s, y compris à l'éminent centre de recherche qu'est l'Université de Californie à Berkeley, où en 1973, 300 étudiant-e-s s'inscrirent pour les 25 places du premier cours offert sur les auteurs 
femmes 9 . C'était une ère de "pouvoir aux étudiants ». Pendant un temps plutôt bref mais critique pour les women's studies, l'accès des étudiant-e-s aux organes de décision pesa d'un poids immense dans la balance en faveur de l'innovation et contre la tradition.

Alors que des postes de professeurs titulaires étaient créés et que l'impératif de publication s'imposait dans les vies des universitaires féministes, le pouvoir échappa aux étudiants. Un certain nombre de batailles amères pour le pouvoir dans les women's studies eut lieu, opposant les étudiant-e-s et celles qui les défendaient (souvent des enseignantes à mi-temps qui y perdaient également) contre les professeures titulaires. La réussite de l'institutionnalisation menaçait l'ancienne unité des formations féministes contre la tradition.

13 L'éducation supérieure aux États-Unis compte quelque 3000 institutions, privées ou publiques, laïques ou confessionnelles : universités en quatre ans (liberal arts), dites globales qui offrent des diplômes du niveau de la maîtrise, une foule de programmes professionnels ainsi que le baccalaureate ${ }^{10}$ et des universités de recherche qui offrent tout cela, plus le doctorat. Ces institutions sont en général contrôlées localement par des boards of trustees si elles sont privées et par le corps législatif de l'État si elles sont publiques; mais dans la plupart des cas, l'administration et le corps enseignant jouissent d'une relative liberté pour déterminer leurs propres programmes et les conditions d'obtention des diplômes. Ainsi, la lutte entre les partisanes de groupes féministes locaux et les universités pour encadrer et contrôler les women's studies - le conflit dit de l'» autonomie contre l'intégration », conflit si important en Allemagne et d'autres pays -, fut résolu assez tôt aux États-Unis en faveur de cette dernière ${ }^{11}$. En dépit de la rhétorique anti-establishment de nombreuses pédagogues féministes de la première heure, l'ouverture des universités à cette nouvelle discipline, la demande pressante des étudiant-e-s et les immenses ressources potentiellement disponibles dans le système ont favorisé une institutionnalisation rapide. La part des doctorats obtenus par des femmes était en forte augmentation. La pression de l'affirmative action aidait à créer des postes de début de carrière. La présence croissante d'enseignantes féministes permit aux women's studies de se développer régulièrement en dépit de la stagnation générale dans la croissance des universités qui accompagna les tendances démographiques et les révoltes des contribuables dans les années 1980 et 1990. Dès le début également, quelques enseignantes bien installées dans leur carrière offrirent un soutien décisif à des moments clés.

La légitimité acquise par les women's studies résulte aussi de la contribution - vitale dans les premiers temps - des fondations privées, en particulier de la Fondation Ford, qui, en commençant par Stanford et Wellesley en 1974, aida au financement de douzaines de centres de recherche, et contribua à la création du journal Signs, de la National Women's Studies Association, et d'autres entreprises professionnelles d'importance. La fondation Woodrow Wilson apporta un appui majeur en 1973 en prenant l'initiative d'un programme d'aide financière pour les thèses dans cette nouvelle discipline. Le travail de Laurel Thatcher Ulrich, lauréate du Prix Pulitzer et aujourd'hui professeure d'histoire à l'Université de Harvard, commença avec une bourse de la fondation Woodrow Wilson pour sa recherche sur la vie d'une sage-femme de la Nouvelle-Angleterre. Selon Ulrich, l'obtention de cette bourse "était une reconnaissance non seulement personnelle mais pour l'entreprise elle-même, c'est-àdire, une prise en compte totale de l'histoire des femmes ${ }^{12}$ ». 
Questions programmatiques et conceptuelles

En combinant les argumentations féministes avec une recherche académique fondée sur les expériences des femmes, ce que les pionnières créèrent, bien au-delà de leurs premiers rêves, ne fut rien d'autre qu'un nouveau champ du savoir. Ses buts étaient étendus; il ne s'agissait pas seulement de transformer l'éducation mais aussi les institutions, pas seulement les consciences mais aussi la société. L'étendue des intérêts académiques féministes est évidente dans le contenu de ses programmes. Alors que les premiers plans de cours, échangés entre les unes et les autres, regroupés et publiés par de nouvelles entreprises féministes au début des années 1970, mettaient surtout l'accent sur l'histoire et la littérature, de nombreux cours existaient dans les sciences sociales, en particulier en psychologie, en sociologie et en anthropologie, ce qui fournissait l'occasion d'études transculturelles comparant les expériences des femmes dans différents contextes. Avec les historiennes, les anthropologues développèrent certains des premiers cadres conceptuels de ce qui fut appelé la théorie féministe. En histoire, selon une étude des articles publiés dans quelque 800 journaux pendant les années 1980, parmi 40 catégories thématiques, les deux plus étudiées étaient le travail ( $14 \% \mathrm{du}$ total) et la religion ( $8 \%)$. Presque un quart de tous les articles $(23 \%)$ se concentrait sur des sujets non américains. Juste derrière en troisième place, venaient les cultures matérielle et populaire et la politique. Les études de la sexualité arrivaient à $6 \%$, alors que la théorie comptait pour $5 \%{ }^{13}$. Même si des chiffres comparables ne sont pas disponibles pour les années 1990, il est probable que la distribution se soit modifiée sensiblement, suivant un intérêt croissant pour la culture des femmes, le corps, la théorie et des sujets liés à la « différence ».

Les choix des étudiant-e-s révèlent une disparité entre les intérêts de la recherche et les effectifs des cours. Depuis le milieu des années 1970 jusqu'à nos jours, les étudiante-s ont fait preuve d'une préférence marquée pour des classes ayant l'étiquette de la "psychologie des femmes" et autres sujets similaires. Un sondage effectué en septembre 2000 auprès des étudiant-e-s inscrits dans le programme que je dirigeais dans les années 1970 ne montra aucun changement à cet égard. La psychologie est depuis longtemps un choix favori de spécialisation parmi les étudiant-e-s du campus. Les départements de psychologie, cependant, sont notoirement rétifs aux études féministes, amenant ainsi de nombreux étudiant-e-s à se tourner vers les women's studies pour apporter un complément à leur travail. Les women's studies en économie et en géographie, disciplines dans lesquelles bien moins de femmes se spécialisent, attirent des effectifs moindres. Des cours en women's studies apparaissent aujourd'hui également dans beaucoup, sinon la plupart, des départements en lettres et sciences sociales. Des matières professionnelles comme la pédagogie, les études d'infirmière et les affaires se sont dans l'ensemble moins ouvertes aux études féministes, peut-être parce que leurs programmes sont hautement recommandés par des agences de qualification.

17 Aux États-Unis, la croissance rapide des women's studies est liée à l'autonomie des universités et des professeurs. Il n'y a pas de ministère de l'Éducation ou autre agence fédérale décidant du recrutement des enseignants ou du contenu des cours. À l'exception de l'établissement d'exigences minimales, les règlements d'éducation des États n'interviennent pas non plus dans les programmes. En outre, l'idée généralement acceptée que les étudiants doivent passer les deux premières années de leurs études supérieures à consolider leurs connaissances en arts et lettres assure des effectifs 
fournis dans les classes de lettres et sciences sociales qui sont les plus à même d'être influencées par les études féministes. Une "éducation générale », en fait, fournit un lieu idéal à l'introduction d'approches critiques à ces disciplines; l'enseignement d'une «pensée critique » est souvent cité comme l'un de ses buts.

En 1984, 71 établissements aux États-Unis offraient des diplômes dans les women's studies en tant que telles (et non pas en tant que spécialisations individualisées ou comme ajouts à d'autres diplômes). En 1997, ce chiffre atteint $248^{14}$. Une spécialisation type requiert une douzaine de cours, y compris un survol introductif qui recouvre diverses expériences de femmes et perspectives féministes; des options tirées d'un choix d'études disciplinaires, de même qu'un cours obligatoire sur les différences transculturelles entre femmes, un autre sur la théorie; et un séminaire chapeautant le tout qui peut exiger un mémoire ou un projet. Approximativement la moitié des cours est offerte sous une rubrique interdisciplinaire, en général les "women's studies ». Le reste est fait dans des départements disciplinaires traditionnels, où une grande majorité de professeures féministes enseignent. Pour rester fidèles aux origines des women's studies issues de l'action locale, et pour répondre au désir persistant des enseignantes et des étudiant-e-s militantes de maintenir des liens avec des féministes hors du monde enseignant, certains programmes offrent et/ou exigent un cours qui se déroule sur le terrain, sous la forme d'un projet de recherche, d'un stage ou d'une autre forme d'» apprentissage d'un service».

19 Cependant, le fait qu'il n'y ait aucun programme standardisé dans les women's studies est une source de satisfaction pour certains, de consternation pour d'autres. Elles ont grandi spontanément, irrégulièrement, suivant les nominations accidentelles de professeures ayant l'intérêt, l'énergie, le bagou et l'influence pour obtenir le soutien à des initiatives féministes académiques. L'idéologie, en particulier l'opposition à l'autorité institutionnelle qui imprégnait les cercles libéraux et féministes dans les années 1960 et 1970, a encouragé une préférence pour une agrégation lâche de cours sous la houlette d'une "coordinatrice ", et une réticence à l'idée de départements dirigés par une «directrice». Cette attitude semble être en train de disparaître de façon inversement proportionnelle à la titularisation des universitaires spécialistes de women's studies et au besoin d'indépendance qui assure un pouvoir de décision en matière de recrutement des professeurs, de détermination des programmes et de programmation des cours.

20 L'institutionnalisation a aussi été retardée par la dépendance des women's studies à l'égard des enseignantes à mi-temps, vacataires ou temporaires. Sans ces éducatrices féministes, les women's studies n'auraient pas pu décoller comme elles l'ont fait - et peut-être pas du tout. Ainsi, lorsque j'ai obtenu un poste de professeur à plein temps mais temporaire en 1974, mes cours ne pouvaient compter pour le diplôme qu'en tant qu'options en women's studies, mais pas dans le département d'histoire (bien que je fusse pleinement qualifiée dans ce domaine). Le département d'histoire engagea ses premières historiennes des femmes plus de dix ans plus tard. Je fus l'une de celles qui eurent de la chance et qui plus tard obtinrent un poste permanent; mais beaucoup d'enseignantes féministes hautement qualifiées furent lésées quand les women's studies gagnèrent leurs premiers postes permanents, surtout si elles s'étaient consacrées à la construction des programmes plutôt qu'à allongement de leurs listes de publications ${ }^{15}$.

Questions institutionnelles et conceptuelles 
21 Les structures et priorités institutionnelles, les politiques suivies par les campus et les personnalités ont provoqué un développement éclaté des women's studies. Il était clair dès le début, cependant, qu'une seule discipline ou une seule méthode ne pourraient suffire à interpréter les expériences et perspectives de la moitié de l'humanité. Nous avons déclaré à une conférence en Californie du Sud en 1978: «Les women's studies sont partout». Vingt ans plus tard, ce que cela signifiait pour un groupe d'universitaires cherchant à identifier quelques-uns des fondements des women's studies, était de l'ordre du « partout est nulle part ${ }^{16}$ ».

22 "Savoir indiscipliné », les women's studies perturbent leurs ami-e-s comme leurs ennemi-e-s. En tentant d'assembler un programme à partir de professeures féministes employées sur son campus dans des départements différents, la théoricienne politique Wendy Brown eut la conviction que les women's studies étaient « impossibles ». Elles avaient beau étendre les limites de leur discipline, les huit professeures (cinq étant formées en littérature ou en histoire, une en théorie politique occidentale, et une en biologie moléculaire) participant au projet dans son université relativement petite, s'aperçurent qu'elles ne pouvaient « combler l'écart entre les intérêts des étudiants et le nôtre ». Mais Brown fut davantage préoccupée par ce qu'elle appela la «formidable fracture des women's studies en tant que domaine de recherche ces dix dernières années », faisant référence aux attaques sur l'emploi du mot "femmes » comme concept fondateur ${ }^{17}$.

Depuis à peu près 1990, les women's studies ont fait face à des défis continuels venus de l'intérieur même du milieu féministe académique. Des livres comme Inessential Woman par Elizabeth Spelman et Am I That Name? par Denise Riley, tous deux publiés en 1988, fournirent une base théorique qui intensifia l'effet d'accusations portées par des lesbiennes ou des femmes de couleur affirmant qu'elles ne se reconnaissaient pas dans la plupart des travaux entrepris ${ }^{18}$. Après une dizaine d'années pendant lesquelles l'accent fut mis sur les différences plutôt que sur les points communs, beaucoup ont éprouvé des difficultés à s'appuyer sur un concept construit socialement et réputé instable comme «la femme» ou «les femmes». D'autres trouvèrent que l'interdisciplinarité tant vantée des women's studies masquait l'échec du dépassement des frontières disciplinaires et ne produisait que de la «multidisciplinarité », et même une sorte de «bricolage intellectuel » (selon la spécialiste de littérature Susan Stanford Friedman ${ }^{19}$ ).

24 Ces deux types de critiques apparaissent aux frontières actuelles du mouvement des women's studies aux États-Unis, à savoir le fait de renommer ou restructurer les programmes féministes académiques sous un chapeau étiqueté « gender studies », et la création de programmes de troisième cycle en women's studies interdisciplinaires. Un enseignement au niveau doctoral fut offert dès le début avec l'inauguration en 1970 d'un programme de troisième cycle à l'Union Institute, qui utilisait des professeures réparties dans tout le pays. En 1972, le Sarah Lawrence College dans l'État de New York créa un programme de maîtrise en histoire des femmes, tandis que l'université californienne de San Jose State élabora une maîtrise en sciences sociales avec un accent sur les women's studies et l'Université de George Washington, dans la capitale fédérale, en initia un en administration publique. Dès 1994, plus de 60 établissements offrirent des diplômes du niveau de la maîtrise, quelque 14 en women's studies et le reste reliant les women's studies à d'autres programmes d'études ${ }^{20}$. 

diplômes propres à certaines disciplines, comme le Ph.D. en histoire des femmes lancé à l'Université de Binghamton dans l'État de New York en 1974, aient suscité un nombre considérable d'inscriptions. Au milieu des années 1990, cependant, l'enseignement interdisciplinaire au niveau doctoral semblait en être à sa phase de décollage. Des programmes existaient dans les universités de Clark dans le Massachusetts, de Emory en Géorgie (toutes deux des institutions privées) et dans celle de York en Ontario au Canada (ainsi qu'un diplôme de Doctor of Arts en Africana Women's Studies à Clark Atlanta University en Géorgie qui date de 1982). En 1999 le nombre avait plus que doublé, avec des programmes dans les campus-phares d'universités de recherche en Iowa, dans le Maryland, le Minnesota, le Michigan et l'État de Washington. D'autres étaient en instance de création ou attendaient d'être approuvés. Le doctorat interdisciplinaire fut le sujet d'un colloque international à l'Université de York en 1995 et le thème d'un numéro de Feminist Studies en $1998^{21}$.

Les arguments pour ou contre le Ph.D. posent la question des ressources; mais même avec le soutien adéquat, l'idée que les professeurs soient cantonnés aux disciplines offrant un diplôme prétendu interdisciplinaire amène des universitaires féministes à s'interroger. Des solutions diverses ont été envisagées pour résoudre ce problème. Elles consistent notamment à exiger un ancrage profond dans une des disciplines, ou même l'admission dans le programme à travers l'une d'elle, comme à Emory; à requérir l'étude de larges zones liées entre elles comme le langage, la littérature et les arts, ou l'histoire, la psychologie et la société, ou la géographie, l'économie et le développement, comme à Clark, ou, comme un nouveau programme à Rutgers University dans le New Jersey, qui cherche à créer un curriculum «vraiment interdisciplinaire » en inventant des champs doctoraux comme «Agency, Subjectivity and Social Change ", " Technology and the Politics of Gender ", " Gendered Borders and Changing Boundaries $»^{22}$.

L'Université de Washington souligne son emplacement au bord de la côte Pacifique en centrant son curriculum sur des études multiculturelles, multiraciales et comparatives. $\mathrm{Au}$ contraire, le modèle collaborateur de l'Université de Toronto fournit aux étudiants un diplôme disciplinaire " renforcé par une 'mention' de leur spécialisation en women's studies». Sa force repose entre autres sur un vaste corps enseignant (plus de 90 professeurs), réputé « briller par la pureté de leur recherche disciplinaire... [qui suscite] un respect immédiat ». On dit que les étudiant-e-s profitent de cette situation en évitant la condition de «sans abri » associée, pour certains, aux programmes autonomes, ainsi que la «teinte» de l'affiliation à un féminisme militant et les soucis liés à l'adaptation de leur diplôme au marché dans le cadre de la compétition à l'emploi ${ }^{23}$.

Il est difficile de savoir jusqu'où va le désir d'interdisciplinarité des étudiant-e-s. L'interdisciplinarité, comme Friedman le souligne, recouvre un champ large et en changement perpétuel, dont la signification n'est pas claire. L'interdisciplinarité estelle « un mélange de disciplines dans lequel les différences sont maintenues, ou une fusion intégrée de différentes disciplines dans quelque chose d'entièrement neuf ? ${ }^{24}$ " L'interdisciplinarité requiert plus qu'une relation additionnelle; c'est plutôt ce que Julie Thompson Klein décrit comme une "triangulation active en profondeur, en largeur et en synthèse ${ }^{25}$ ». Elle nécessite, selon Judith Allen et Sally Kitch, toutes deux directrices de programmes de women's studies, des «analyses à un métaniveau de 
l'épistémologie des disciplines ${ }^{26}$ ». À mon sens, ce besoin est plus facilement atteint dans des programmes de Ph.D. de women's studies ${ }^{27}$.

Jusqu'à présent, l'interdisciplinarité est rarement posée comme concept ou processus. Comme beaucoup de débats intellectuels, celui-ci reflète l'histoire des institutions locales. Le concept de «discipline» est aussi construit socialement et variable. Cette réalité est souvent masquée par son association avec le "département», unité puissante du gouvernement académique. En général, les départements attribuent les ressources et confèrent des récompenses. Ils ont tendance à fonctionner comme des " nations » isolées, indépendantes, protégeant même agressivement leurs frontières et prérogatives. La sociologue Barrie Thorne, faisant allusion à Marx et Weber, les décrit comme des "systèmes de travail académique [...], des fiefs, des baronnies, où des hommes importants construisent des empires ». Dans ce schéma, les partisanes des women's studies sont les nouveaux - quoique plus aimables - «barbares [...] qui portent nos stigmates comme des boucliers plutôt que de se cacher comme celles qui les ont précédés, dans l'économie domestique ${ }^{28}$ ".

En tant qu'unités de gouvernements, cependant, les départements peuvent ressembler à l'empire austro-hongrois plus qu'à l'Autriche contemporaine; c'est-à-dire que beaucoup d'entre eux n'ont pas de frontières clairement délimitées, de méthodologie ou de langue unique ou singulière, de mission de recherche pure et simple. Quand Brown se plaint de la multiplicité des techniques et des sujets parmi lesquels les étudiants de thèse doivent choisir et que les professeurs doivent enseigner; quand Friedman se lamente sur le «trop » qui défie l'ampleur du domaine de n'importe quel enseignant, elles ignorent les choix que les étudiants et les enseignants dans d'autres champs doivent faire ${ }^{29}$. La dispersion est beaucoup plus extrême (et plus analogue aux women's studies) en anthropologie et en biologie. J'ai appris un jour que l'anthropologie n'était pas considérée comme une discipline dans tous les pays, mais plutôt comme un domaine interdisciplinaire comprenant la paléontologie, la primatologie, l'archéologie, la linguistique, et autres. Mais personne, à ma connaissance, ne prétend que le Ph.D. en anthropologie est «impossible» ou « incohérent». La biologie comprend de nombreuses subdivisions à la fois dans ses branches cellulaires et moléculaires.

31 Il y a de vraies raisons, qui peuvent parfois être prohibitives, pour ne pas se lancer dans un programme indépendant de Ph.D. en women's studies. Mais elles sont le reflet, à mon sens, de soucis pragmatiques liés à la politique, aux pratiques, aux ressources, et vraisemblablement aux personnalités aussi. En outre, les étudiant-e-s comme les enseignantes qui s'engagent dans le féminisme académique risquent une perte de pouvoir, de prestige, de relations amicales et d'argent. Les départements traditionnels confèrent ce que Renate Klein appelle un "privilège disciplinaire » à leurs membres ${ }^{30}$. Ils peuvent aussi fournir une excellente formation, une identité professionnelle, des collègues avec qui l'on souhaite travailler. Il y a clairement de bonnes raisons pour lesquelles les postes disciplinaires ou à cheval sur deux départements, en dépit de leurs inconvénients, continuent d'attirer beaucoup d'universitaires spécialistes de women's studies.

Les gender studies

Des considérations intellectuelles, politiques et personnelles sont aussi impliquées dans la tendance actuelle visant à remplacer les women's studies par les gender studies comme le fief du féminisme académique. J'ai appris, pendant les douze ans passés à être 
doyenne et vice-présidente d'université, supervisant des dizaines de programmes académiques, qu'il n'est pas facile de séparer les intérêts et les courants intellectuels qui embrouillent les fils du discours universitaire. Ce fut évident dans les témoignages de six participantes à la table-ronde sur "What's in a Name?" ("Que cache un nom?») lors de la réunion annuelle de la National Women's Studies Association en $1999^{31}$. Trois raisons dominantes émergèrent. D'après elles, ces nouveaux noms semblent 1) plus généraux et notamment mieux à même d'inclure les hommes et la masculinité, les gay/lesbian et queer studies ; 2) plus scientifiques et théorisables ; 3) moins ouvertement politiques (c'est-à-dire féministes). Cette tendance n'est pas nouvelle, puisque le Center for Research on Women de l'Université de Stanford, fondé en 1974, est devenu un Institute for Research on Women and Gender en 1986. Le Program for the Study of Women and Men in Society à l'Université de Californie du Sud (USC), fondé en 1975, fut renommé Gender Studies en 1997-98. D'autres exemples abondent.

Le problème lié au fait de renommer, comme celui lié à l'interdisciplinarité et à la structure institutionnelle, s'est posé dès le début, quand les women's studies, avec leur apostrophe qui brouille les différences entre études des, par, sur ou pour les femmes, ont commencé à être populaires ${ }^{32}$. La dichotomie faite aujourd'hui entre "study of women » et « women's studies » émergea, à mon avis, à cause des problèmes, présentés plus haut, liés aux départements et aux disciplines. La différence entre "femmes » et "genre » reflète plus que tout l'émergence de la théorie féministe telle qu'elle a été transmise dans le milieu académique féministe américain par Joan Scott, Denise Riley, Judith Butler et d'autres, souvent lourdement influencées par des théoriciens français et fréquemment associée aux départements d'anglais ou de littérature ${ }^{33}$. L'autre défi majeur que pose l'emploi du terme "femme» apporté par les femmes de couleur, aurait pu être plus facilement géré en élargissant les catégories et la vision de manière à comprendre la pluralité et la diversité de la vie des femmes. Il est ironique de constater que, alors que les gender studies semblent plus conservatrices (quand on fait abstraction des rapports de pouvoir), moins associées au féminisme (que certains regardent maintenant comme si c'était un mot obscène) et au militantisme en dehors des cercles académiques, la minorité qui connait le travail radical déconstructionniste de Butler et de ses adeptes réalise que le genre implique une attitude révolutionnaire à l'égard des rôles sexuels traditionnels et des valeurs sociales. À l'instar des queer studies, il permet de chapeauter toutes les formes de la théorie et de la pratique qui transgressent les normes sexuelles. Il peut aussi être utilisé pour souligner la nature systématique de la subordination des femmes ${ }^{34}$. Du fait que c'est un terme qui défie toute définition simple, il remplit plusieurs objectifs.

L'évolution vers les gender studies reflète aussi l'insatisfaction suscitée par les women's studies dont les progrès limités n'ont pas permis de satisfaire les objectifs originels de transformation. Scott, par exemple, attribue partiellement son attirance initiale pour les questions théoriques à «sa frustration face à l'impact relativement limité que les women's studies avaient sur les études d'histoire en général ${ }^{35}$ ». Ce que j'appelle "la quête de théorie » reflète aussi, à mon sens, la prise de conscience par les féministes académiques dans les années 1970 que «la théorie, perçue traditionnellement comme masculine, semblait occuper une place privilégiée dans le monde académique, et que, si nous voulions compter pour quelque chose, nous autres dans les women's studies nous devions en avoir un peu». Nous en avions aussi besoin pour expliquer l'histoire de l'oppression des femmes et pour montrer des voies de résistance et de changement. 
Ceci explique peut-être partiellement pourquoi un groupe non négligeable et influent d'universitaires féministes fut attiré par le socialisme qui offrait non seulement une promesse de justice sociale mais aussi un corpus théorique bien défini qui semblait utile pour expliquer l'oppression historique des femmes. Ce groupe, déjà un peu familiarisé avec la philosophie européenne, fut attiré par les idées poststructuralistes européennes, précisément alors que le marxisme échouait visiblement à expliquer de manière adéquate la condition des femmes ${ }^{36}$.

Dans les women's studies, où l'enjeu de la mise au jour de « la construction sociale du deuxième sexe " vit le jour dès 1968, les débats sur le mot « femme » qui allaient jusqu'à la négation de sa validité conceptuelle tout en soulignant les relations entre « sexe » et "genre » et le fonctionnement du pouvoir, ne pouvaient qu'échouer à étancher la soif de théorie de ce domaine ${ }^{37}$. L'analyse de genre promet de rendre aux women's studies ce que la germaniste Biddy Martin appelle "une bonne partie de sa vigueur intellectuelle», vigueur dont elle déplore la perte dans son domaine de women's studies. Martin espère aussi voir les gender studies traverser les frontières entre les différents champs du savoir, spécialement vers les sciences naturelles comme la neurobiologie ${ }^{38}$. L'historienne Leora Auslander, parlant depuis l'Université de Chicago, qui résista à la vague jusque dans le milieu des années 1990 puis créa un Center for Gender Studies, explique comment, à cette date tardive, après l'essor des women's studies et des gay/lesbian studies dans le monde académique, il semblait " rétrograde " de créer des programmes séparés ${ }^{39}$.

Auslander et Brown posent leurs arguments sur des bases à la fois intellectuelles et pratiques. Le but exprimé à Chicago est de fournir « un centre où tous ceux travaillant sur le genre et la sexualité seraient les bienvenus ». On y est très conscient aussi que « les gender studies risquent de devenir des white studies » (Auslander). Aussi y a-t-on développé les travaux sur les différences de " race » et d'ethnicité. Ces élargissements successifs ne devraient-ils pas mener à la création d'un "Centre sur l'étude des différences » ? Joan Scott, creusant ce thème dans une entrevue avec deux thésards de Brown University, examine l'interdisciplinarité dans ses relations avec le Ph.D. en women's studies. S'inspirant du concept de Stuart Hall selon lequel «la théorie provoque le glissement du sens alors que la politique fige une signification précise dans un but particulier " (concept qui leur fut transmis par Wendy Brown), ces étudiants tentèrent d'envisager une université idéale pour un doctorat interdisciplinaire en women's studies. Mais, comme le dit Scott, «ce n'est pas comme ça qu'on travaille ${ }^{40}$ ». Ce commentaire à l'emporte-pièce est en fait très éclairant ; il explique en effet qu'une grande part de la controverse sur les women's studies et les gender studies, comme tant d'autres choses dans le monde académique, dépend précisément de "comment on travaille ». L'efficacité est souvent l'atout de la théorie.

Alors que certaines théoriciennes désignent le "genre» comme une étiquette unificatrice et utile pour héberger le féminisme académique, des universitaires plus enclines à des études empiriques ou à l'activisme politique, résistent. Le genre, selon elles, escamote les femmes. Il dissimule les femmes en tant qu'objets d'étude ou sujets dans la société. Il suggère une égalité fallacieuse entre les sexes, non seulement comme catégories d'analyse, mais aussi comme êtres humains dans le monde. De plus, celles qui défendent l'intitulé "women's studies » nient qu'il ne soit pas englobant. «Les women's studies sont intrinsèquement des gender studies » d'après Beverly GuySheftall, directrice et fondatrice des «black women's studies » à Spelman College. C'est 
aussi l'opinion de Shirley Yee, directrice des women's studies à l'Université de Washington. Toutes deux sont des femmes de couleur, qui sont gênées non par l'absence du genre, mais par celle des questions de classe et de race. Pour Guy-Sheftall, une évolution vers des gender studies ou des feminist studies réduirait, plutôt qu'il n'agrandirait, l'horizon des women's studies. Pour Yee, « le mot 'femmes' dans women's studies, certes problématique, est en fait plus englobant comme point focal de l'analyse que le nom ne le suggère, et est nécessaire comme stratégie politique dans le système universitaire ». Elle trouve que «le moment où le débat éclate est plus dérangeant que le débat lui-même ", le moment où les women's studies ont élargi leur horizon pour inclure des groupes de femmes historiquement marginalisés. Selon Yee, les women's studies analysent nécessairement le genre alors que l'inverse n'est pas forcément vrai. À l'instar des ethnic studies, les women's studies sont «enracinées dans des mouvements politiques spécifiques et historiques qui ne peuvent pas facilement être escamotés ${ }^{41} »$. Il est tellement facile de priver les femmes - et l'histoire des femmes - de toute considération...

38 À mon sens, on ne doit ni oublier ni se lamenter sur les origines politiques des women's studies. Ceci constitue un argument fort en faveur du maintien du nom, seul ou combiné avec gender. Les historiennes du féminisme savent que la perte de mémoire historique, récurrente, retarde l'accomplissement des objectifs féministes. L'histoire des women's studies montre de la même manière que la mémoire institutionnelle des efforts d'une génération risque de disparaitre si elles sont subsumées sous une plus large rubrique. Ce que le fait d'obtenir des postes de titulaires dans les women's studies a garanti - ou semblait avoir garanti jusqu'à maintenant - est que les revendications et les conquêtes engendrées par le féminisme de la deuxième vague soient gardées dans la langue et la littérature de la vie universitaire. Cela devait servir à créer des cohortes d'étudiant-e-s entrés dans les classes sans conscience féministe mais en sortant souvent avec une.

Quelles étudiant-e-s?

Où les étudiant-e-s (et les étudiants) se situent-elles dans les affrontements qui assaillent le féminisme académique aujourd'hui ? Les femmes représentent aujourd'hui environ $60 \%$ des étudiants de second cycle dans le supérieur aux États-Unis, environ 40 \% des effectifs de troisième cycle et de formation professionnelle. Les étudiants en women's studies sont en majorité des femmes. Maints rapports mentionnent entre 85 et $90 \%$, bien que le contingent masculin puisse atteindre un tiers dans certains cas. Il semble aussi que les hommes sont plus nombreux à s'inscrire quand les women's studies apparaissent avec une dénomination plus large. Le Program for the Study of Women and Men in Society de l'Université de Californie du Sud et le Gender Studies Program de Lewis and Clark College dans l'Oregon déclarent tous deux avoir une proportion relativement plus grande d'hommes prenant leurs cours ${ }^{42}$.

Autant souligner tout de suite un point crucial : les étudiant-e-s ne s'identifient pas initialement comme des «féministes». Ceci n'est pas simplement le reflet des connotations négatives associées depuis peu au féminisme; j'avais noté ce fait dès $1978^{43}$. Pour beaucoup, je pense, les women's studies répondent à la quête de ce qu'une génération antérieure appelait « une philosophie de la vie ${ }^{44} »$. Ce n'est pas, comme on l'en accuse parfois, simplement une recherche d'identité personnelle dans le sens étroit que ce terme suggère aujourd'hui. Ces étudiant-e-s témoignent fréquemment de 
l'impact que les women's studies ont sur leurs esprits et leurs vies, ce qui est corroboré par les données de recherche.

41 Une évaluation à l'échelle nationale de ce que les étudiant-e-s apprennent dans les women's studies, menée au début des années 1990, confirme les impressions que l'enseignement dispensé est rigoureux et amène les étudiant-e-s à penser de façon critique. "Dans les cours de women's studies, on soumettait une idée à notre évaluation. J'ai appris à mettre en question des choses que je lisais pour la première fois. On donnait notre opinion, mais on nous demandait aussi des preuves » dit une étudiant-e. Une autre déclara que les women's studies « s'ouvrent par des questions... C'est vraiment cela la plus grande différence... On met tout en question, tout le temps, tout le temps. »Elles facilitent aussi l'intégration de l'intellect et de l'affect. Les étudiant-e-s disent et redisent : «cela a changé ma vie ${ }^{45}$ ». Mise au défi d'expliquer à quoi son diplôme en women's studies et en religious studies lui servira, une femme dit: "Je vais penser ${ }^{46}$ ». Comme la plupart de ceux qui se spécialisent dans les lettres, les étudiant-e-s se concentrant sur les women's studies choisissent de ne pas suivre la foule de ceux pour qui des objectifs de carrière sont capitaux. Et leur nombre grandit. Selon le ministère fédéral de l'Éducation, de 119 baccalaureates décernés en 1986-87, on est passé à 411 en 1992-93 ${ }^{47}$.

Dans la première enquête nationale complète sur les diplômés en women's studies, d'anciennes élèves ont fait part du défi intellectuel que représente un diplôme dans les women's studies, mais aussi de l'utilité d'un tel diplôme. Un plan d'études qui exigeait "le courage de mettre en question" apporta en retour la confiance en soi et l'ingéniosité. Plus de la moitié des sondées poursuivaient leurs études dans le troisième cycle. Celles qui étaient employées occupaient des emplois allant de responsable syndicale à pilote de ligne. Beaucoup travaillaient dans des professions d'assistance. Beaucoup avaient aussi fait preuve de leur initiative en se façonnant des emplois non traditionnels pour elles-mêmes ${ }^{48}$.

Mon impression personnelle, après avoir rencontré l'an dernier des spécialistes en women's studies dans des séminaires de trois universités différentes (Emory University, SDSU et Stanford), après avoir écouté leurs présentations dans des réunions nationales, et lu leurs publications, est que l'un des meilleurs arguments en faveur de ce domaine est la qualité de ses étudiant-e-s.

L'intérêt des enseignants

44 L'impact des women's studies sur les premières enseignantes est bien étudié. Plusieurs recueils d'essais autobiographiques décrivent l'émergence des women's studies dans la vie et le travail de celles et ceux qui furent les pionniers de ce domaine en histoire, littérature et sociologie. De nouvelles questions, de nouveaux concepts et catégories d'analyse, des interprétations revues et corrigées de la sagesse traditionnelle ont surgi à la croisée des liens entre ce qu'elles étudiaient et les perspectives extérieures qu'elles amenaient à leur discipline ${ }^{49}$.

Mais elles ont aussi "transgressé » les frontières de leur domaine d'origine pour emprunter des vues féministes d'ailleurs. Judith Allen et Sally Kitch ont compilé une liste des plus grands concepts innovateurs dans les women's studies qui sont largement appliqués au-delà de leurs disciplines d'origine. On compte parmi eux la «voix différente» de Carol Gilligan, le «genre performatif» de Judith Butler, la déconstruction de "l'égalité par opposition à la différence " de Joan Scott, et bien d'autres. Et pourtant, dans l'ensemble, les efforts sont encore insuffisants pour faire des 
comparaisons à l'intérieur même des disciplines ou pour analyser comment les débats d'un domaine peuvent apparaitre sous la lumière d'un autre. Par exemple, la " preuve de l'expérience » de Scott a des implications bien au-delà de l'histoire, mais, d'après Allen et Kitch, n'a pas voyagé bien loin ${ }^{50}$. À mon avis, l'» originalité déplacée » et l'ancrage dans l'histoire de la théorisation par des universitaires entretenant peu de rapports avec des disciplines voisines, restent des problèmes ${ }^{51}$.

L'ouverture des différentes disciplines au savoir féministe est très variable. Elle est considérable dans des domaines comme l'histoire, la littérature ou l'anthropologie, mais plutôt modeste en économie, sciences politiques et philosophie ${ }^{52}$. En 1995, une enquête conduite sous les auspices de la National Women's Studies Association sur les administratrices de programme montra que seulement $8 \%$ avaient des postes exclusivement en women's studies, et que $15 \%$ avaient des postes mixtes. La grande majorité appartenait donc, institutionnellement, aux disciplines traditionnelles. De plus, $65 \%$ des programmes et départements n'avaient pas à leur disposition de postes d'enseignement à plein temps ${ }^{53}$. Une autre étude sur 72 féministes académiques dans six établissements de recherche montra que la plupart s'identifiaient à une seule discipline et en étaient contentes. Leur but est d'intégrer le savoir féministe dans leur domaine d'origine ${ }^{54}$. Étant donné les problèmes qu'éprouvent les enseignantes ayant des postes mixtes (confrontés à une double loyauté) et le rôle de l'argent (en particulier les fonds accordés par les départements pour la recherche), l'attractivité des disciplines va sans doute continuer à bloquer les women's studies dans les marges des universités américaines ${ }^{55}$.

L'impact sur le monde universitaire et la société

Néanmoins, de nouveaux programmes sont créés chaque année. On ne peut évidemment qu'estimer l'ampleur du changement causé par les women's studies dans le monde académique; c'est la question du verre à moitié vide ou à moitié plein. Les conditions de pluralisme et de décentralisation qui ont facilité leur développement sont précisément celles qui rendent difficile une généralisation de ses effets. Puisque des études empiriques sur une grande échelle ne sont pas encore disponibles, il faut bien s'appuyer sur des observations individuelles. La philosophe Martha Nussbaum, par exemple, souligne les nouvelles perspectives apportées par les women's studies aux théories philosophiques de la justice et aux théories économiques de distribution des biens ; aucune des deux n'avait considéré la famille comme une institution concernée par les questions de justice et d'injustice ${ }^{56}$.

L'historienne Ruth Rosen crédite aussi les women's studies d'un impact majeur sur les institutions éducatives. Dans son étude sur l'impact du militantisme féministe moderne sur l'histoire américaine, Rosen écrit : «Les féministes ont fondamentalement modifié les curricula des écoles, des colleges et des universités américaines de façon à ce que dès le cours préparatoire, aucun enfant ne puisse encore s'imaginer que les femmes n'avaient ni travaillé, ni élevé des familles, écrit, aimé, ne s'étaient battues, avaient protesté et s'étaient organisées dans le passé. Entre 1970 et 1990, une révolution a eu lieu dans les institutions d'enseignement supérieur américaines ${ }^{57}$ ».

Pourtant, Rosen s'est plainte ailleurs du manque d'attention prêté au savoir féministe. "C'est comme si, dit-elle, les féministes avaient construit un jardin gargantuesque au milieu de la ville, que seuls quelques habitants ont remarqué ${ }^{58}$ ". Des universitaires de premier plan peuvent encore ignorer le travail intellectuel d'une génération de féministes, même dans leur propre discipline, sans pour autant être discrédités ou 
considérés comme dépassés. La persistance d'une telle ignorance fut démontrée en 1997 dans une série d'essais publiés par des universitaires distingués dans Daedalus, le journal de l'Académie américaine des Arts et des Sciences. Dans ce passage en revue de cinquante ans de "transformation" dans quatre disciplines majeures, à savoir l'économie, la littérature, la philosophie et les sciences politiques, les women's studies étaient à peine mentionnées ${ }^{59}$. Quand elles sont traitées dans la presse populaire, les women's studies apparaissent sous la caricature de militantes féministes défilant en bottes sur les campus, ou comme un sanctuaire de l'extrémisme du "politically correct». Une image déformée peut être tellement envahissante qu'un critique a récemment commencé un article très laudateur sur l'expérience d'une anthropologue enseignant les women's studies par ces mots: "Si vous n'êtes pas un fan du genre si péniblement politiquement correct connu sous le nom de women's studies... » avant de passer à ses louanges sur le livre ${ }^{60}$.

La presse abonde également en concepts et en termes qui ont vu le jour dans le savoir féministe et ont affecté la façon dont les gens pensent et dont les législateurs agissent dans la vie quotidienne. Depuis la «féminisation de la pauvreté» jusqu'au " harcèlement sexuel », la recherche débutée ou développée dans les women's studies s'est «fait pousser des jambes» et est allée se promener. Ce sont les universitaires féministes qui ont fourni la documentation sur le travail des femmes et ont calculé la valeur économique des tâches ménagères, des soins aux enfants et autres " corvées de l'amour ». Il reste plus facile, cependant, de rendre naturelles les différences sexuelles que de comprendre le processus à travers lequel les identités de sexe et de genre se sont développées, se sont maintenues, et ont été contestées. La longue histoire de la domination masculine dans les institutions sociales, y compris dans nos vénérables universités, suggère que ce nouveau savoir et ces innovations éducatives initiées par les women's studies ne vont pas être facilement acceptées ou assimilées.

51 Pour conclure, toutefois, sur deux notes positives, citons, d'abord, une récente étude nationale selon laquelle $36 \%$ des enseignants estiment que « beaucoup » de cours ayant un contenu féministe sont délivrés dans leurs établissements ${ }^{61}$. Enfin, le président de la Fondation Woodrow Wilson, dans la newsletter de la fondation (diffusée largement sur Internet), déclare: "Les women's studies sont devenues un modèle en matière d'interdisciplinarité... [Elles] ont fourni ce que nous tous devons valoriser: des organisations du savoir authentiquement fraîches. [Elles sont] devenues un modèle pour questionner des frontières trop rigides, pour libérer la question indiscutable qui appelle les combinaisons d'expertise souvent surprenantes pour lui répondre... [Les women's studies] ont accompli le travail de plusieurs siècles en quelques dizaines d'années ${ }^{62} \%$.

52 Aujourd'hui, la croissance la plus rapide des women's studies a lieu en dehors de ses régions d'origine. Un livret international publié en 1993 dressait la liste de plus de cent pays où des cours ou des centres de recherche en women's studies, des programmes de formation spécialisés pour les femmes ou des publications avaient vu le jour ${ }^{63}$. Dans la propagation des women's studies à chaque continent et dans l'application de la recherche féministe aux problèmes des femmes dans les pays développés et en voie de développement, les women's studies enrichissent à la fois le fonds du savoir humain et contribuent à la « recherche concrète de la justice entre les sexes ${ }^{64}$ ».

53 Traduction par Yves Figueiredo ${ }^{65}$ 


\section{BIBLIOGRAPHIE}

« Women's Studies on the Edge », 1997, differences 9, no 3, fall.

Allen Ann Taylor, 1993, « The March through the Institutions : Women's Studies in the United States and West and East Germany, 1980-1995 », Signs, 22, n 1, fall : 152-180.

Allen Judith A., Kitch Sally L., 1998 « Disciplined by Discipline ? The Need for an Interdisciplinary Research Mission in Women's Studies », Feminist Studies, 24, n 2, summer : 275-299.

Armatage Kay, 1999, « Collaborating on Women's Studies : The University of Toronto Model », Feminist Studies, 24, $\mathrm{n}^{\circ}$ 2, summer : 347-355.

Auslander Leora, 1997, « Do Women's + Feminist + Men's + Lesbian and Gay + Queer Studies = Gender studies ? », differences, $9, \mathrm{n}^{\circ} 3$, winter : 1-30.

Beverly Evelynn M., 1997, « Whither Black Women's Studies. Interview », differences, 9, n 3, winter : 31-45.

Boris Eileen, Chaudhuri Nupur eds., 1999, Voices of Women Historians : The Personal, the Political, and the Professional, Bloomington, Indiana University Press.

Bowles Gloria, Klein Renate Duelli, eds., 1983, Theories of Women's Studies, New York, Routledge and Kegan Paul.

Bowles Gloria, s.d., « Living Ideas : A Personal/Political Chronicle of Berkeley Women's Studies, 1973-1985 », manuscrit non publié.

Boxer 2000, « Unruly Knowledge : Women's Studies and the Problem of Disciplinarity », NWSA Journal, 12, n² , summer : 119-129.

Boxer b, 1998, «Remapping the University : The Promise of the Women's Studies Ph.D. », Feminist Studies, $24, \mathrm{n}^{\circ} 2$, summer : 387-402.

BOXER Marilyn J., 1978, « Closeup : Women's Studies Department at San Diego », Women's Studies Newsletter, 6, $\mathrm{n}^{\circ} 2$, spring : 20-23.

--, 1982, « For and About Women : The Theory and Practice of Women's Studies in the United States », Signs, 7, n 3, spring : 660-695.

--, 1989, « Women's Studies, Feminist Goals, and the Science of Women », in Carol R. Pearson, Donna L. Shavlik, and Judith G. Touchton eds., Educating the Majority: Women Challenge Tradition in Higher Education, New York, Macmillan : 184-204.

--, 1998, When Women Ask the Questions : Creating Women's Studies in America, Baltimore, MD, Johns Hopkins University Press.

Brown Loulou et al. eds., 1993, The International Handbook of Women's Studies, New York, Wheatsheaf.

Brown Wendy, 1997, « The Impossibility of Women's Studies », differences, 9, n 3, fall : 79-101. Butler Judith, 1993, Gender Trouble : Feminism and the Subversion of Identity ; New York, Routledge. Butler Judith, Scott Joan W. eds., 1992, Feminists Theorize the Political, New York, Routledge. Chamberlain Mariam ed., 1998, Women in Academe : Progress and Prospects, New York, Russell Sage Foundation. 
Chamberlain Mariam K., Bernstein Alison, 1992, «Philanthropy and the Emergence of Women's Studies », Teacher's College Record 93, $n^{\circ}$ 3, spring.

Chronicle of Higher Education Almanach, 2000, Issue 47, $n^{\circ} 1,1 / 9$.

Cook Kathryn, Henry Renea avec Scott Joan W., 1997, « The Edge. Interview », differences, 9, nº 3, winter : 132-155.

Farnham Christie, 1992, « Foreword », in Gayle V. Fischer ed, Journal of Women's History: Guide to Periodical Literature, Bloomington, Indiana University Press.

Feminist Studies 1998, 24, n² , summer.

Friedman Susan Stanford, 1998, « (Inter)disciplinarity and the Question of the Women's Studies Ph.D. », Feminist Studies 24, n² 2, summer : 301-325

Greene Gayle, Kahn Coppélia eds., 1993, Changing Subjects : The Making of Feminist Literary Criticism, London et New York, Routledge.

Hoff Joan, « Introduction : An Overview of Women's History in the United States », Journal of Women's History : Guide : 9-11.

Hoffman Frances L., Stake Jayne E., 1998, « Feminist Pedagogy in Theory and Practice : An Empirical Investigation », NWSA Journal, 10, $\mathrm{n}^{\circ}$ 1, spring : 79-97.

Howe Florence ed., 2000, The Politics of Women's Studies : Testimony from Thirty Founding Mothers, New York, Feminist Press.

Kidd Karen, Spencer Ande eds., 1994, Guide to Graduate Work in Women's Studies, 2nd ed., NWSA, University of Maryland, College Park.

KLEIN Julie Thompson, 1990, Interdisciplinarity : History, Theory, and Practice, Detroit, Wayne State University Press.

--, 1996, Crossing Boundaries : Knowledge, Disciplinarities, and Interdisciplinarities, Charlottesville, University Press of Virginia.

Laslett Barbara et Thome Barrie eds., 1997, Feminist Sociology : Life Histories of a Movement, New Brunswick, N.J., Rutgers University Press.

Lorber Judith, 1994, Paradoxes of Gender, New Haven, Yale University Press.

Lorde Audre, 1984, « The Master's Tools Will Never Dismantle the Master's House », Sister Outsider : Essays and Speeches, Freedom, Calif., Crossing Press.

Luebke Barbara F., Reilly Mary Ellen, 1995, Women's Studies Graduates : The First Generation, New York, Teacher's College Press.

Martin Biddy, 1997, « Success and Its Failures », differences, 9, n³, winter : 102-131

Musil Caryn McTighe ed., 1992, The Courage to Question : Women's Studies and Student Learning, Washington, DC, Association of American Colleges et National Women's Studies Association.

Nussbaum Martha C., 1997, Cultivating Humanity : A Classical Defense of Reform in Liberal Education Cambridge, Harvard University Press.

Nussbaum Martha, 2000, Women and Human Development : The Capabilities Approach, Cambridge University Press.

O’Barr Jean, Wyer Mary eds. 1994, Engaging Feminism : Students Speak Up and Speak Out, Charlottesville, University Press of Virginia. 
Orr Catherine M., 1999, « Tellings of Our Activist Past : Tracing the Emergence of Women's Studies at San Diego State College ", Women's Studies Quarterly, 27, $\mathrm{n}^{\circ}$ 3-4, fall : 212-229.

Riley Denise, 1988, « Am I That Name? » Feminism and the Category of « Woman » in History, Minneapolis, University of Minnesota Press.

ROSEN Ruth, 1999, « Secrets of the Second Sex in Scholarly Life » in Chronicle of Higher Education, 30/9.

,-- 2000 , The World Split Open : How the Modern Women's Movement Changed America, New York, Viking.

SCOTT Joan W., 1986, « Gender : A Useful Category of Historical Analysis », American Historical Review $91, \mathrm{n}^{\circ}$ 5, october : 1053-1075.

,-- 1988, Gender and the Politics of History, New York, Columbia University Press.

Scully Diana, 1996, « Overview of Women's Studies : Organization and Institutional Status in US Higher Education », NWSA Journal, 8, $\mathrm{n}^{\circ}$ 3, fall : 122-128.

Shteir Ann B. ed., 1996, Graduate Women's Studies : Visions and Realities, North York, Ontario, Inanna Publications.

SPELMAN Elizabeth V., 1988, Inessential Woman : Problems of Exclusion in Feminist Thought, Boston, Beacon Press.

Weisbuch Robert, 1999, « Why Women's Studies? », Woodrow Wilson National Fellowship Foundation Newsletter, Fall.

Women's Studies Quarterly, 1997, 25, n 1-2, spring-summer : 422.

YEE Shirley J., 1997, « The 'Women’ in Women's Studies », differences, 9, n 3, winter : 46-64.

--, 1999, « Establishing an International Doctoral Program in Women's Studies at the University of Washington », Feminist Studies, 24, n 2, summer : 366-373.

\section{NOTES}

1.Pour une étude récente du développement des women's studies aux États-Unis, voir Boxer 1998. Pour une histoire détaillée des premiers développements et des références bibliographiques les plus précoces, Boxer 1982.

2.« Women's Studies on the Edge » 1997.

3.En raison de problèmes de définition, il est délicat et peut-être peu fiable de compter les thèses en women's studies; mes chiffres s'appuyent sur les titres définis comme tels par le personnel à University Microfilms à Ann Arbor, Michigan. La différence entre les chiffres s'explique par des diplômes de maitrise, décernés en grande partie dans des établissements canadiens où un travail de recherche de ce niveau est considéré comme étant comparable aux thèses américaines. Voir Boxer 1998 : 241-242, 312 n. 3.

4.Ibid. : 26-27.

5.Pour un résumé de la législation prohibant la discrimination sexuelle dans les études supérieures, voir Chamberlain 1998, spécialement le chapitre 2.

6. "Women's Studies », Newsweek, 26 octobre $1970: 61$. D'autres cours sur les femmes étaient apparus bien plus tôt ; par exemple un cours de la fin du xixe siècle par la sociologue Sophonisba Breckinridge à l'Université de Chicago ; l'historienne Eleanor 
Flexner sur « la question de la femme » à New York en 1953-54; un cours sur la féminologie enseigné par Mme Souley-Darqué à Paris en 1901.

7.Les effectifs en histoire tombèrent de 44000 à 21000 entre 1972 et 1979 ; Farnham $1992: 3$.

8.L'expression « outils du maître » est généralement employée négativement, pour se référer à des méthodes conventionnelles rejetées par de nombreux intellectuels féministes ; je suis en désaccord avec cette vue. Voir Lorde 1984.

9.Bowles s.d. : 9.

10.Le baccalaureate est un diplôme concluant un programme d'études de quatre ans après le Secondaire.

11.Allen 1993. Pour une étude de cas qui est un bon exemple du divorce entre le campus et la communauté qui eut lieu à SDSU la première année des women's studies, voir Orr 1999.

12.Parmi les autres agences de financement, on compte les fondations Carnegie, Rockefeller, Russell Sage et Charles Stewart Mott, de même que la National Endowment for the Humanities et autres agences publiques. En 1992, Ford avait donné 22 des 36 millions de dollars en fonds privés attribués au soutien des women's studies;

Chamberlain, Bernstein 1992 : 556-558. Pour Ulrich, voir Robert Weisbuch 1999.

13. Hoff s.d.

14. Chamberlain 1998 : 137 ; Women's Studies Quarterly 1997 : 422.

15.Voir, par exemple, Bowles s.d.

16.Pour les women's studies comme « savoir indiscipliné», voir Boxer 2000. Pour

l'» impossibilité », voir Brown $1997: 82$.

17.Ibid., 83.

18.Riley 1988 ; Spelman 1988.

19.Friedman $1998: 312$.

20.Pour une liste complète, quoique maintenant dépassée, des 111 programmes de troisième cycle, voir Kidd, Spencer 1994.

21.Shteir 1996 et Feminist Studies 1998.

22.Rapporté par Abena P.A. Busia, conférence sur "Women's Studies Then and Now » du CUNY Graduate Center, 2 décembre 2000.

23.Pour le programme de l'Université de Washington, voir Yee 1999 ; sur le programme doctoral de Toronto, avec quelque 60 étudiants, voir Armatage 1999 : 349-350.

24.Friedman 1998.

25.L'analyse la plus complète de l'interdisciplinarité se trouve dans l'œuvre de Klein 1990 et 1996. Sur la « triangulation », voir Klein 1996: 212.

26.Allen, Kitch 1998 : 278.

27. Voir Boxer 1998 b.

28. Notes de l'auteure, Conférence de la Fondation Spencer sur les « Women's Studies and the Study of Women ", Chicago, 1/10/1998.

29.Brown W. $1997: 84$; Friedman 1998 : 314.

30.Klein, commentaire fait en session à la réunion annuelle de la NWSA, Albuquerque, Juin 1999 sur l'ouvrage de Bowles et Klein 1983.

31.Les membres du panel représentaient Jersey City State University, Louisiane State University, Kenyon College, North Carolina State University, Sonoma State University, et Yale University.

32.Pour une traitement plus complet des noms, voir Boxer $1998: 13,257 \mathrm{n}^{\circ} 12$.

33.Scott 1986 ; Scott 1988 ; Riley 1988, Butler 1993 ; Butler, Scott 1992. 
34. Voir Lorber 1994.

35.Scott $1988: 13$

36.Boxer $1998: 13,126$.

37." The Social Construction of the Second Sex ", article de Jo Freeman écrit en 1968, fut publié en 1970 et souvent réimprimé. « Psychology Constructs the Female, or the Fantasy Life of the Male Psychologist », de Naomi Weisstein, date aussi de 1968 (Cf. Boxer 1998 : 284 pour les données de première publication).

38.Martin 1997 : 102.

39.Auslander 1997 : 3.

40.Cook, Henry, Scott 1997 : 147-148.

41.Guy-Sheftall, Hammonds $1997: 41$; Yee 1997 : 48-49, 56.

42.Pour Lewis and Clark, où $41 \%$ des sondés lors d'un sondage des anciens élèves étaient des hommes, voir Musil $1992: 46$.

43. Boxer 1978 : 20-23.

44.Boxer 1998 : 96-97.

45.Cette discussion se base sur Musil 1992 ; voir Boxer 1998 : 92-93. Pour des propos d'étudiants, voir aussi Luebke, Reilly 1995 et O’Barr, Wyer 1994.

46.Boxer 1989 : 184.

47. Pour un diagramme des diplômes en women's studies décernés annuellement entre 1986-87 et 1992-93, voir Boxer 1998 : 260-261, nº 8.

48.Luebke, Reilly 1995.

49.Laslett, Thome 1997 ; Greene, Kahn 1993 ; Boris, Chaudhuri 1999 ; Howe 2000.

50.Allen, Kitch 1998 : 284-286.

51.Merci à Catherine Stimpson pour l'expression « originalité déplacée » (displaced originality).

52.Commentaires de Louise Lamphere, professeure d'anthropologie à l'Université du Nouveau Mexique, à l'époque president-elect de l'American Anthropology Association, et de Sylvia Yanigisako, professeure d'anthropologie à l'université de Stanford, à la conférence de la Fondation Spencer, Chicago, 1/10/1999. Mes commentaires sur les autres domaines sont fondés partiellement sur leur revue dans Daedalus (hiver 1997) et partiellement sur mon expérience en tant qu'administratrice de deux grandes universités urbaines en Californie.

53.Scully 1996.

54.Lynne Goodstein, en session sur « The Challenge of Success : Strategies for Ensuring the Future of Women's Studies in the 21st Century ", conférence annuelle de la NWSA, Albuquerque, juin 1999.

55.Les données démographiques sur les enseignants en women's studies sont rares. Une petite enquête récente, entreprise dans le cadre d'une étude comparative sur les pratiques pédagogiques dans les cours de women's studies et des autres matières dans 7 universités du Midwest entre 1990 et 1993 mit en avant les caractéristiques suivantes : sur 115 sondés, 87 \% s'identifièrent de sexe féminin, $88 \%$ de race blanche (sic), avec un âge moyen de 43 ans, $51 \%$ avaient un poste de maître de conférence ou de professeur (en général titulaire) et $79 \%$ enseignaient dans les lettres ou les sciences sociales ( $7 \%$ en sciences physiques, $15 \%$ dans des domaines professionnels). Voir Hoffman, Stake 1998 : 85.

56.Nussbaum $1997: 196$.

57.Rosen $2000:$ 265-266.

58.Rosen 1999 : A48. 
59.Pour de plus amples développements, Boxer 1998 : 241-242.

60.Martha Beck, « Recombinations : An Anthropologist Explores the New Social Relations Wrought by Modern Culture », critique de Mary Catherine Bateson, Full Circles, Overlapping Lives : Culture and Generation in Transition (New York : Random House, 2000) dans New York Times Book Review, 28/5/2000, p. 12.

61.Étude (1998-99) par le Higher Education Research Institute de UCLA, rapportée dans le Chronicle of Higher Education Almanach $2000: 40$.

62.Weisbuch 1999.

63.Brown et al. 1993.

64.Cette expression est empruntée à Nussbaum 2000 : 303.

65.L'auteure remercie le Marilyn Yalom Fund de Stanford University qui a financé la traduction de cet article.

\section{RÉSUMÉS}

Ces trente dernières années, les women's studies se sont développées aux États-Unis et sont devenues un nouveau domaine du savoir dans l'enseignement supérieur. Entre 1978 et 1995, plus de 10000 étudiants ont obtenu un diplôme doctoral fondé sur un travail de recherche dans ce domaine. Les women's studies ont amené de nouvelles questions, de nouveaux concepts, de nouvelles catégories d'analyse et des interprétations révisées de la sagesse conventionnelle à beaucoup - si ce n'est à la plupart - des disciplines littéraires et dans les sciences sociales. Cependant, les women's studies doivent faire face à de grands défis, tant conceptuels que d'organisation. Les critiques mettent en question la validité du concept «femme " comme concept fondateur, préférant soit subsumer les women's studies dans le " gender ", soit même placer l'étude des femmes et du « gender » dans les disciplines traditionnelles.

During the past thirty years women's studies in the United States has grown to constitute a new field of knowledge in higher education. Between 1978 and 1995, more than 10000 scholars earned doctoral degrees for research categorized as women's studies. This field has brought new knowledge to many - if not most - disciplines in the humanities and social sciences. Nevertheless, women's studies today faces serious challenge from critics who question the validity of "woman » as its a foundational concept and prefer to subsume " women's studies » into "gender studies» or even to confine studies of women and gender to the traditional disciplines.

\section{AUTEUR}

\section{MARILYN BOXER}

Marilyn J. Boxer est professeure d'histoire à San Francisco State University et collabore à l'Institute for Research on Women and Gender à Stanford University. Elle dirigea de 1974 à 1980 le premier programme de women's studies à San Diego State University, où elle devint ensuite doyenne de la Faculté des Arts et des Lettres. Suivirent alors sept années comme vice-présidente des études à San Francisco State University. Elle est l'auteure de When Women Ask the Questions : 
Creating Women's Studies in America (1998) et co-auteure de Connecting Spheres : European Women in a Globalizing World, 1500 to the Present, 2e édition (2000). Elle a aussi écrit sur les femmes socialistes au début de la Troisième République. 